\title{
Hospital accreditation pounds another 'nail' in the 'smoking' coffin
}

As regular readers of the JAOA will recall, we have consistently called for a smokeless society. We have encouraged the American Osteopathic Association (AOA), the colleges of osteopathic medicine, the osteopathic hospitals, and individual osteopathic physicians to join the cause against smoking by leading the way through example.

We have had the pleasure of reporting the progress made in the establishment of "smokefree" campuses at many of the colleges of osteopathic medicine. We have told you of the numerous osteopathic hospitals that have restricted or have prohibited smoking in their facilities. Even the central offices of the AOA became "smoke-free" more than 1 year ago. Yet, we can and must do much more in the battle against the number 1 cause of cardiovascular and pulmonary disease and cancer among Americans.

An example of this added effort occurred at the annual convention, held this year in New Orleans. The AOA Board of Trustees approved the recent revision of the Accreditation Standards of the AOA Committee on Hospital Accreditation. The previous policy issued by the committee required that hospitals establish a smoking policy that permitted smoking in designated areas only. The new policy limits patient smoking to strict circumstances. Furthermore, the new policy reads as follows:

The hospital must have, disseminate, and enforce a hospital-wide no-smoking policy.

A. The policy shall prohibit smoking and the use of tobacco products by hospital patients, medical staff, hospital employees, visitors, and volunteers within the hospital buildings.

B. Hospitals may develop medical exceptions to their policy, but only for patients currently under treatment at the facility where abrupt cessation of smoking would be detrimental to the patient.

1. The policy shall prohibit smoking by patients unless authorized to smoke by the attending physician.

2. The hospital will segregate, where practical, patients authorized to smoke from nonsmoking patients.

This strengthened accreditation standard represents another "nail" in the "smoking" coffin.

THOMAS WESLEY ALLEN, DO Editor in Chief

\section{Changing of the guard at the AOA}

"To remain young one must change," wrote Alexander Chase in Perspectives. As the American Osteopathic Association prepares to celebrate a centennial of service to America, change is taking place at our helm. John P. Perrin, executive director since 1982 , has assumed the duties of president of the University of Health Sciences College of Osteopathic Medicine (UHS-COM) in Kansas City, Mo.

As the search committee seeks an appropriate replacement for Mr Perrin, AOA President Gilbert S Bucholz, has assumed the duties of acting executive director.

During Mr Perrin's tenure as executive director, the profession has grown substantially in number and in strength. Although this growth was the result of a concerted effort by many, it was Mr Perrin's leadership that provided the catalyst for our advancement.

As the first non-DO executive director, $\mathrm{Mr}$ Perrin helped to improve the unity of the profession by promoting open dialogue among the divisional societies and the practice affiliates. With the augmentation of the internal public relations department, Mr Perrin played a vital role in enhancing the public's understanding - and acceptance - of osteopathic medicine. 
This public awareness has reached new heights with the ongoing Care-A-Van project.

Administratively, the purchase of the AOA central headquarters building during Mr Perrin's tenure has provided the association and its members with a long-term facility to accommodate further growth. The building, located just off Chicago's Magnificent Mile, has enhanced the profession's image as well.

More than just improving our image, $\mathrm{Mr}$ Perrin worked to improve membership services. Under his direction, a new professionalism emerged among the AOA staff. A director of membership services was hired and the finance department was reorganized. Computerized membership services and the addition of electronic publishing equipment are just two examples of the upgraded facilities that streamline day-to-day operations.

Certainly, Mr Perrin has done much more during his almost-quarter-century association with osteopathic medicine than can be enumerated here. In fact, as president of UHS$\mathrm{COM}, \mathrm{Mr}$ Perrin will continue to have a hand in shaping the profession's future.

As we await the arrival of a new executive director who will lead osteopathic medicine into its second century as well as the 21 st century, we look back on the past decade with pride and gratitude to $\mathrm{Mr}$ Perrin for his outstanding leadership and continued commitment to the profession.

THOMAS WESLEY ALLEN, DO Editor in Chief

\section{Accepting applications for}

\section{Executive Director of the American Osteopathic Association, Chicago, Ill.}

Send narrative letter on why you feel qualified for this position, plus current résumé or $\mathrm{CV}$.

Qualified DOs and non-DOs may apply.

Send application, in confidence, to Frank J. McDevitt, DO, chairman, AOA Search Committee, 18593 Jamestown Road, Northville, MI 48167-1832.

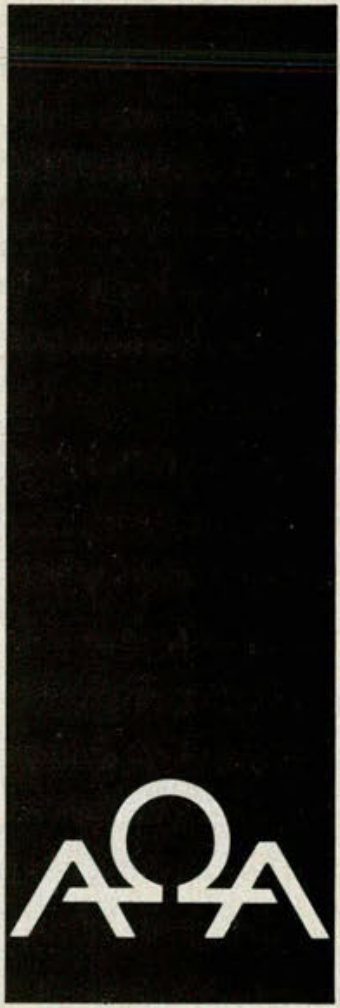




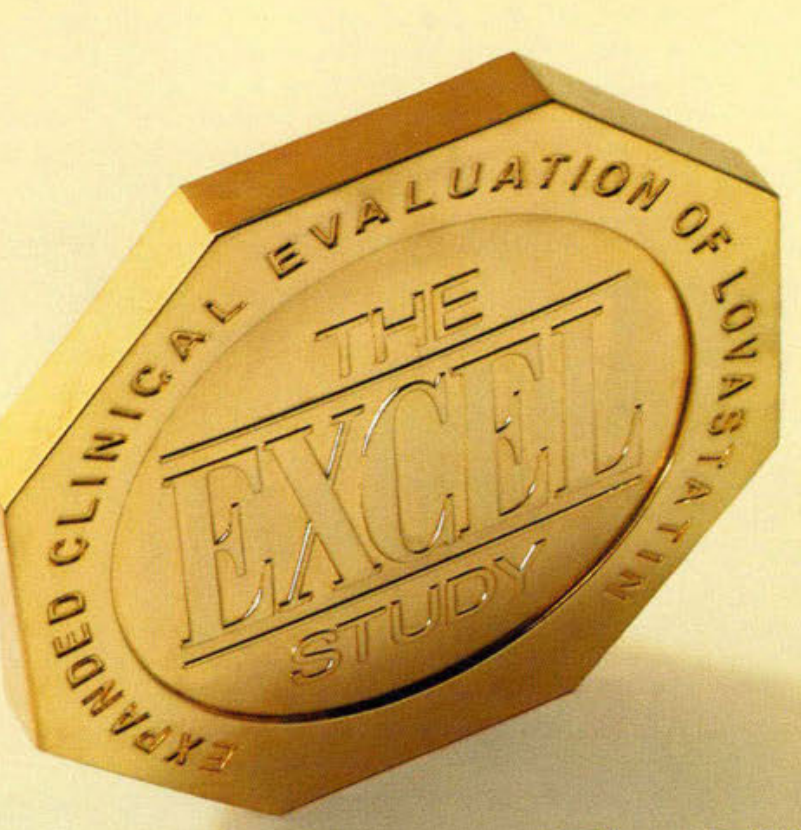

The EXCEL Study:

48 weeks, 8,245 patients, 362 medical centers, in the largest-ever U.S. randomized, double-blind, placebo-controlled trial of patients with primary hypercholesterolemia

the result e are in...

For a Brief Summary of Prescribing Information

please see the last page of this advertisement. 

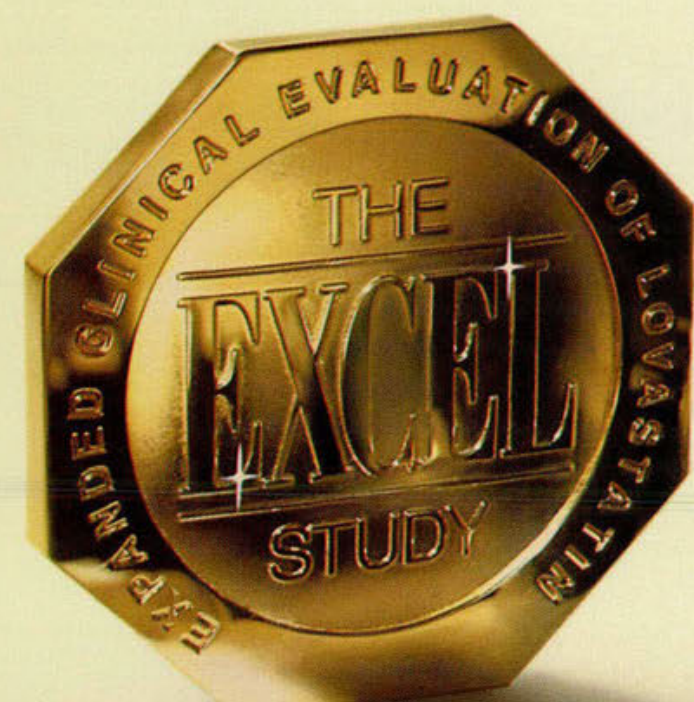

Lovastatin lowere

\section{Lovastatin lowere}

\section{Lovastatin lowere}

\section{Lovastatin raisec}

MEVACOR ${ }^{\otimes}$ (Lovastatin, MSD) is contraindicated in patients who are hypersensitive to any component of the medication; in patients with active liver disease or unexplained persistent transaminase elevations; in pregnant or lactating patients; and in women of childbearing age, except when such patients are highly unlikely to conceive.

For complete details on MEVACOR, including cautionary information regarding myopathy, drug interactions, and liver enzyme monitoring, please refer to the Prescribing Information.

For a Brief Summary of Prescribing Information, please see the last page of this advertisement.

"Mean percent changes from baseline $(n=6,582)$

**Triglyceride values are median changes.

Dosage ranged from $20 \mathrm{mg}$ q.p.m. to $40 \mathrm{mg}$ b.i.d.

Copyright ( $\odot 1991$ by MERCK \& CO., INC. 


\section{DL cholesterol $24-40 \%$}

\section{tal cholesterol $17-29 \%$}

\section{iglycerides $10-19 \%$}

\section{IDL cholesterol $6.6-9.5 \%$}

For many patients with primary hypercholesterolemia (Types Ila and Ilb), when diet and other nondrug therapies are inadequate
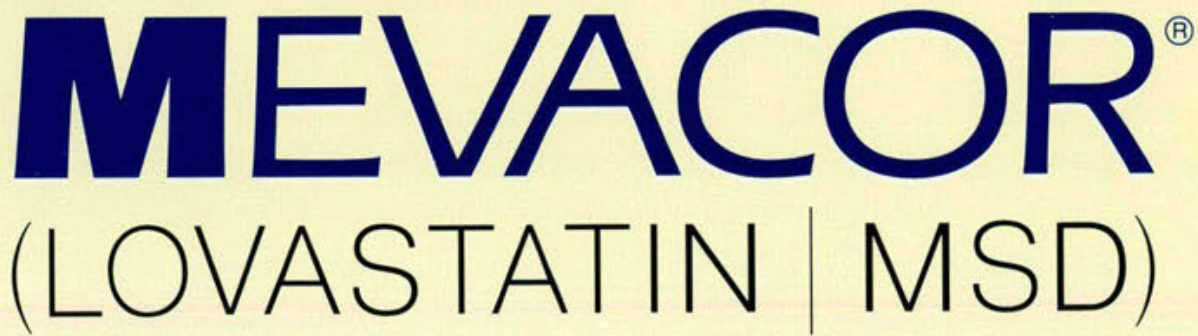

MSD) 
CONTRAINDICATIONS: Hypersensitivity to any component of this Active liver disease or unexplained persistent elevations of serum Pregnancy and lactation.

lowering drugs during pregnancy should have little impact on the outcome of long-term therapy of grimary terol and other products of the cholesterol biosynthesis pathway are essential components for fetal development, including synthesis of steroids ductase such as MEVACOR* (Lovastatin, MSD) to decrease the synthesis of cholesterol and possibly other products of the cholesterol biosynthesis pathway, MEVACOR may cause fetal harm when administered to a pregnant woman. Therefore, lovastatin is contraindicated during pregnancy. Lovastatin should be administered to women of childbearing age only pregnant while taking this drug. lovastatin should be discontinued and the patient should be apprised of the putential hazard to the fetus.

WARNINGS: Liver Dysfunction: Marked persistent increases (to more than 3 times the upper limit of normal) in serum transaminases occurred in $1.9 \%$ of adult patients who received lovastatin for at leasi one year in clinical trials (see ADVERSE REACTIONS). When the drug usually fell slowly to pretreatment levels. The increases usually appeared associated with jaundice or other clinical signs or symptoms. There was no evidence of hypersensitivity. It is recommended that liver function tests be performed during ther. apy witt lovastatin. Serum transaminases, including ALT (SGPT), should be monitored before treatment begins, every 6 weeks for the first 3 months, every 8 weeks during the remainder of the first year, and periodically thereafter (e.g., at approximately 6 -month intervals). Spetransaminase levels, and in these patients, measurements should be re peated promptly and then performed more frequently. If the transaminase upper limit of normal and are persistent, the drug should be discontinued. discontinuation of the drug

The drug should be used with caution in patients who consume substantial quantities of alcohol and / or have a past history of liver disease Active liver disease or unexplained transaminase elevations are contrain dications to the use of lovastatin.

As with other lipid-lowering agents, moderate (less than 3 times the upper limit of normal) elevations of serum transaminases have been reported following therapy with MEVACOR (see ADVERSE REACTIONS) These changes appeared soon after initiation of therapy with MEVACOR
were often transient, were not accompanied by any symptoms, and interwere often transient, were not accompa
ruption of treatment was not required.

Skeletal Muscle: Rhabdomyolysis has been associated with lovastatin therapy alone, when combined with immunosuppressive therapy including cyclosporine in cardiac transplant patients, and when combined in non-transplant patients with either gemfibrozil or
lipid-lowering doses ( $\geq 1$ g day) of nicotinic acid. Some of the affected patients had preexisting renal insufficiency, usually as a consequence of long-standing diabetes. Acute renal failure from rhabdomyolysis has and has also been reported in transplant patients receiving lovastatin plus cyclosporine.

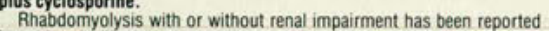
in seriously ill patients receiving erythromycin concomitantly with erythromycin should be carefully monitored

Fulminant rhabdomyolysis has been seen as early as 3 weeks after initiation of combined therapy with gemfibrozil and lovastatin but may be seen after several months. For these reasons, it is felt that, in mosi subjects who have had an unsatisfactory lipid response to either drug
alone, the possible benefits of combined therapy with lovastatin and alone, the possible benefits of combined therapy with lovastatin and
gemfibrozil do not outweigh the risks of severe myopathy, rhabdo gemfibrozil do not outweigh the risks of severe myopathy, rhabdo
myolysis, and acute renal failure. While it is not known whether this interaction occurs with fibrates other than gemfibrozil, myopathy an rhabdomyolysis have occasionally been associated with the use of other fibrates alone, including clofibrate. Therefore, the combined use of lovastatin with other fibrates should generally be avoided.

Physicians contemplating combined therapy with lovastatin and
pont lipid-lowering doses of nicotinic acid or with immunosuppressive drugs should carefully weigh the potential benefits and risks and should care. fully monitor patients for any signs and symptoms of muscle pain, apy and during any periods of upward dosage titration of either drug. Periodic CPK determinations may be considered in such situations, bi there is no assurance that such monitoring will prevent the occurrence of severe myopathy. The monitoring of lovastatin drug and metabolite
levels may be considered in transplant patients who are treated with
immunosuppressives and lovastatio.

Lovastatin therapy should be temporarily withheld or discontinued in hy patient with an acute serious condition suggestive of a myopathy any patient with an acute, serious condition suggestive of a myopathy or secondary to rhabdomyolysis, including severe acute infection, hypotension, major surgery, trauma, severe metabolic

electrolyte disorders, and uncontrolled seizures.
Myalgia has been associated with lovastatin therapy. Transient, mildly

Myalgia has been associated with lovastatin therapy. Transient, mildly
elevated creatine phosphokinase levels are commonly seen in lovastatin-

elevated creatine phosphokinase levels are commonly seen in lovastatinpatients developed a myopathy, i.e. myalgia or muscle weakness sidered in any patient with diffuse myalgias, muscle tenderness, or weakness, and or ply ticularly if accompanied by malaise or fever. Lovastatin therapy should be ficularly if accompanied by malaise or fever. Lovastatin therapy should be
discontinued if markedly elevated CPK levels occur or myopathy is diagosed or suspected.

Most of the patients who have developed myopathy (including rhabdomyolysis) while taking lovastatin were receiving concomitant therapy with immunosuppressive drugs, gemfibrozil, or lipid-lowering doses of nico-
tinic acid. In clinical trials, about $30 \%$ of patients on concomitant immunosuppressive therapy including cyclosporine developed myop athy: the corresponding percentages for gemfibrozil and niacin were

In 6 patients with cardiac transplants taking immunosuppressive ther apy including cyclosporine concomitantly with lovastatin $20 \mathrm{mg}$ /day, the elevated to approximately 4 times the expected levels. Because of an ap parent relationship between increased plasma levels of active metabolites derived from lovastatin and myopathy, the daily dosage in patients taking ADMINISTRATION). Even at this dosage, the benefits and risks of using lovastatin in priate diet, exercise, weight reduction in obese patients, and to treat other underlying medical problems (see INDICATIONS AND USAGE). els (see ADVERSE REACTIONS). This should be considered in the differential diagnosis of chest pain in a patient on therapy with lovastatin. Homozygous Familial Hypercholesterolemia: MEVACOR* (Lovastatin, MSD) is less effective in patients with the rare homozygous familial hypercholesterolemia, possibly because these patients have no func-
tional LDL receptors. MEVACOR appears to be more likely to raise gous patients.

Orug Interactions: Immunosuppressive Drugs, Gemfibrozil, Niacin (Nicotinic Acid), Erythromycin: See WARNINGS, Skeletal Muscle.
Coumarin Anticoagulants: In a clinical trial in warfarin-treated patients designed specifically to observe a potential effect of lovastatin on the prothrombin time, lovastatin in dosages up to $40 \mathrm{mg}$ b.i.d. did not produce since the drug was marketed, clinically evident bleeding and/or increased prothrombin time have been reported in a few patients taking coumarin anticoagulants concomitantly with lovastatin. The causal relationship to
lovastatin is unclear. Nevertheless, it is recommended that in patients taking anticoagulants, prothrombin time be determined before starting lovastatin and frequently enough during early therapy to insure that no significant alteration of prothrombin time occurs. Once a stable prothrom-
bin time has been documented, prothrombin times can be monitored at the intervals usually recommended for patients on coumarin anticoagurepeated. Lovastatin therapy has not been associated with bleeding or Antipyrine: Antipyrine is a model for drugs metabolized by the micro-
somal hepatic enzyme system (cytochrome P450 system). Because lovastatin had no effect on the pharmacokinetics of antipyrine, interactions with other drugs metabolized via this mechanism are not expected. pharmacokinetic or pharmacodynamic interaction with concomitant dministration of single doses of lovastatin and propranolo.

Digoxin: In patients with hypercholesterolemia, concomitant adminis-
tration of lovastatin and digoxin resulted in no effect on digoxin plasma Concentrations.
Other Concomitant Therapy: Although specific interaction studies were not performed, in clinical studies, lovastatin was used concomitantly with inflammatory drugs (NSAIDs) without evidence of clinically significant

Carcinogenesis, Mutagenesis, Impairment of Fertility: In a 21-month carcinogenic study in mice, a statistically significant $(p<0.05)$ increase in at doses of $500 \mathrm{mg} / \mathrm{kg} / \mathrm{day}$ ( 312 times the maximum recommended human dose) of lovastatin. These changes were not seen in mice given
doses of 20 and $100 \mathrm{mg} / \mathrm{kg} / \mathrm{day}$ ( 12.5 and 62.5 times the maximum rec-

A statistically significant increase $(p \leq 0.05)$ in the incidence of pulmonary adenomas was seen in female mice receiving $500 \mathrm{mg} / \mathrm{kg} / \mathrm{day}$ ( 312 were seen in males at any dose or in fermales receiving 20 or $100 \mathrm{mg} / \mathrm{kg}$ cause the incidence of pulmonary tumors was within the range of
untreated animals in studies of similar duration, the relationship of this In addition, an increase in the incidence of papilloma in the non-glandular mucosa of the stomach was observed in mice receiving 100 and 500 $\mathrm{mg} / \mathrm{kg} / \mathrm{day}$ ( 62.5 and 312 times the maximum recommended human
dose): no increase was seen at a dosage of $20 \mathrm{mg} / \mathrm{kg} / \mathrm{day}$ ( 12.5 times the dose): no increase was seen at a dosage of $20 \mathrm{mg} / \mathrm{kg} / \mathrm{day}$ ( 12.5 times the
maximum recommended human dose). The glandular mucosa was not tantly, there is a strong association between this change and hyperplasia of the squamous epithelium (acanthosis) in this region; acanthosis is a characteristic change observed in the non-glandular mucosa of rodents of inhibition of the reductase in this tissue.

Similar squamous epithelium is found in the esophagus and anorectal junction of the mouse and rat; however, no evidence of a similar drug: induced hyperplastic response was observed in these tissues in studies of
up to 21 months in the mouse given up to $500 \mathrm{mg} / \mathrm{kg}$ /day ( 312 times the maximum recommended human dose), or in a study of 24 months in the
rat given $180 \mathrm{mg} / \mathrm{kg} /$ day (112 times the maximum recommended human In a 24-month carcinogenicity study in rats, there was a positive dose response relationship for hepatocellular carcinogenicity in males (unad. spontaneously in this strain of rat, the implications of this finding are

Drug-related testicular atrophy, decreased spermatogenesis, spermatocytic degeneration and giant ceil formation were seen in dogs starting at $20 \mathrm{mg} / \mathrm{kg} / \mathrm{day}$ ( 12.5 times the maximum recomm
The clinical significance of these findings is unclear

No evidence of mutagenicity was observed in a microbial mutagen test using mutant strains of Salmonella typhimurium with or without rat of
mouse liver metabolic activation. In addition. no evidence of damage to genetic material was noted in an in vitro alkaline elution assay using rat or genetic material was noted in an in vitro alkaline elution assay using rat or
mouse hepatocytes, a V-79 mammalian cell forward mutation study. an in vitro chromosome aberration study in CHO cells, or an in vivo chromo Nomal aberration assay in mouse bone marrow.
No drug-related effects on fertility were found in studies with rats

Pregnancy: Pregnancy Category $X$ : See CONTRAINDICATIONS Lovastatin has been shown to produce skeletal malformations in the rat
fetus at doses of $800 \mathrm{mg} / \mathrm{kg} / \mathrm{day}(500$ times the maximum recommended
human dose). At similar doses in mice, an increase in skeletal malforma. tions was observed. These individual changes are within the range of those observed spontaneously in this strain of mouse. No drug-induced
changes were seen in either species at doses of up to $80 \mathrm{mg} / \mathrm{kg} /$ day ( 50 changes were seen in either species at doses of up to $80 \mathrm{mg} / \mathrm{kg} /$ day $(50$ mations was noted in rabbits at up to $15 \mathrm{mg} / \mathrm{kg}$ /day (highest tolerated dos data in pregnant women.

Nursing Mothers: Studies in rats have shown that lovastatin is excreted in the milk. It is not known whether this drug is excreted in human milk.
Because many drugs are excreted in human milk and because of the Because many drugs are excreted in human mik and because of the
potential for serious adverse reactions in nursing infants from MEVACOR Women taking lovas
CONTRAINDICATIONS)

Pediatric Use: Safety and effectiveness in children have not been established. Because children are not likely to benefit from cholesterol lowering for at least a decade and because experience with this drug is limited (no
studies in subjects below the age of 20 years), treatment of children with lovastatin is not recommended at this time.

ADVERSE REACTIONS: MEVACOR is generally well tolerated; adverse reactions usually have been mild and transient. Less than $1 \%$ of patients
were discontinued from controlled clinical studies due to adverse experiwere discontinued from controlled clinical studies due to adverse exper-
ences attributable to MEVACOR. About $2 \%$ of patients were discontinued from all studies (controlled and uncontrolled) due to adverse experiences tinued due to increases in serum transaminases.
Clinical Adverse Experiences: Adverse experiences reported in pa are shown in the table below:

$\begin{array}{ccc}\text { MEVACOR Placebo } & \text { Cholestyramine } \\ (\mathrm{N}=613) & (\mathrm{N}=82) & (\mathrm{N}=88)\end{array}$

\begin{tabular}{|c|c|c|c|}
\hline \\
\hline Gastrointestinal & & & \\
\hline $\begin{array}{l}\text { Constipation } \\
\text { Diarthea }\end{array}$ & $\begin{array}{l}4.9 \\
5.5\end{array}$ & $\overline{4.9}$ & $\begin{array}{r}34.1 \\
8.0\end{array}$ \\
\hline Dyspepsia & 3.9 & $\overline{24}$ & $\begin{array}{l}13.6 \\
21.6\end{array}$ \\
\hline Abdominal pain/cramps & 5.7 & 2.4 & 5.7 \\
\hline $\begin{array}{l}\text { Heartburn } \\
\text { Nausea }\end{array}$ & $\begin{array}{l}1.6 \\
4.7\end{array}$ & $\overline{37}$ & 8.0 \\
\hline $\begin{array}{l}\text { Nausea } \\
\text { Musculoskeletal }\end{array}$ & & & \\
\hline Muscle cramps & 1.1 & - & 1.1 \\
\hline $\begin{array}{l}\text { Myalgia } \\
\text { Pychiatric }\end{array}$ & 2.4 & 1.2 & \\
\hline $\begin{array}{l}\text { Nervous System/Psychiatric } \\
\text { Dizziness }\end{array}$ & 2.0 & 1.2 & \\
\hline Headache & 9.3 & 4.9 & 4.5 \\
\hline Rash/pruritus & 5.2 & - & 4.5 \\
\hline $\begin{array}{l}\text { Special Senses } \\
\text { Blurred vision }\end{array}$ & 1.5 & - & 1.1 \\
\hline Dysgeusia & 0.8 & - & 1.1 \\
\hline
\end{tabular}

Laboratory Tests: Marked persistent

About $11 \%$ of patients had elevations of creatine phosphokinase levels of at least twice the normal value on one or more occasion corresponding values for the control agents were cholestyramine, 9 probucol, $2 \%$. This was attributable to the noncardiac fraction of
large increases in CPK have sometimes been reported (see WARN

Concomitant Therapy: In controlled clinical studies in which lova was administered concomitantly with cholestyramine, no adverse tions peculiar to this concomitant treatment were observed. The ad
reactions that occurred were limited to those reported previously vastatin or cholestyramine. Other lipid-lowering agents were not a uncontrolled clinical studies, most of the patients who have devel myopathy were receiving concomitant therapy with immunosuppre
drugs, gemfibrozil, or niacin (nicotinic acid) (see WARNINGS, Sk
Muscle)

Uncontrolled Clinical Studies: The adverse experiences observ uncontrolled studies were similar to those seen in controlled cil
studies. Abnormal liver function tests were observed at a higher incid the controlied studies (see WARNNGS, Liver Dystunction). M athy (myalgia with marked CPK elevations) was reported
mately $0.5 \%$ of patients (see WARNINGS, Skeletal Muscle).

Causal Relationship Unclear: Nervous System: Peripheral neuro has been reported; the relationship to lovastatin is uncertain. phy in over 30 patients showed no evidence of neurotoxic effec
phe

Eye: Lens opacities (see CLINICAL PHARMACOLOGY, Clinical Stuo Post-marketing Experience: Additional adverse experiences occu since the drug was marketed are listed below:

Gastrointestinal: Hepatitis, chronic active hepatitis, chole aundice, fatty change in liver, and, rarely fulminant hepatic necrosis osis, and hepatoma; anorexia, vomiting.

has been reported rarely which has included one or more of the follo features: anaphylaxis, angioedema, lupus-like syndrome, polymy mia, positive ANA, ESR increase, arthritis, arthralgia, urticaria, asth photosensitivity, fever, chills, flushing, malaise, and dyspnea. anxiety; paresthesia. drome: toxic epidermal necrolysis.

Eye: Progression of cataracts (lens opacities)

Estrointestinal: Pancreatitis, stomatitis.

Nervous System/Psychiatric: Depression, insomn

Metabolic: Edema

Clinical Laboratory Test Findings
Liver Function fests: Liver tu

elevated alkaline phosphatase and bilinubin.

Thyroid Function fests: Rare reports of thyroid function test at OVERDOSAGE: The oral LD $D_{50}$ of MEVACOR in mice is $20 \mathrm{~g} / \mathrm{kg}$ Five healthy human volunteers have received up to $200 \mathrm{mg}$ of lovas as a single dose without clinically significant adverse experiences. A
cases of accidental overdosage have been reported: no patients had specific symptoms, and all pa
imum dose taken was 5 to 6

Until further experience is obtained. no specific treatment of over

The dialyzability of lovastatin and its metabolites in man is not kno

DOSAGE AND ADMINISTRATION: The patient should be placed on a dard cholesterol-lowering diet before receiving MEVACOR and sh.
continue on this diet during treatment with MEVACOR. MEVACOR sh be given with meals.

The recommended starting dose is $20 \mathrm{mg}$ once a day given with
evening meal. The recommended dosing range is 20 to 80 molday gle or divided doses; the maximum recommended dose is $80 \mathrm{mg}$ gle or divided doses; the maximum recommented dose is $80 \mathrm{mg}$ Doses should be individualized according to the patient's response
Tables I to IV under CLINICAL PHARMACOLOGY. Clinical Studies for response results)

For those patients with severely elevated serum cholesterol le
e. $>300 \mathrm{mg} / \mathrm{dL}$ [ [7.8 mmol/L] on diet). MEVACOR may be initiated a In patients taking immunosuppressive drugs concomitantly ovastatin (see WARNINGS. Skeletal Muscle), therapy should begin $0 \mathrm{mg}$ of MEVACOR and should not exceed $20 \mathrm{mg}$ day. Cholesterol levels should be monitored periodically and consider
should be given to reducing the dosage of MEVACOR if cholesterol fall below the targeted range.

Concomitant Therapy: Preliminary evidence suggests that the cho terol-lowering effects
tyramine, are additive.

Dosage in Patients with Renal/nsufficiency: In patients with severe
insufficiency (creatinine clearance $<30 \mathrm{~mL} / \mathrm{min}$ ). insufficiency (creatinine clearance $<30 \mathrm{~mL} / \mathrm{min}$ )
dosage increases above $20 \mathrm{mg}$ day should be care mented cautiously (see CLINICAL PHARMACOLOGY mented cautiously (see CLINICA
and WARNINGS. Skeletal Muscle)

$\mathrm{DOH}$

For more detailed information, consult your MSD Representative or Co. INC. West Point PA 19486 . JOMC49R3 